\title{
Interpretação Sísmica e Avaliação de Oportunidades Exploratórias na Área do Pré-Sal, Norte da Bacia de Santos
}

\author{
*Rodrigo Ferro (HALLIBURTON), Gabriela Salomão Martins (FGEL/UERJ), Maria Eliege Gloria (OGX), Carmela Pereira
}

(FGEL/UERJ), Douglas Lisboa (OGX)

Copyright 2016, SBGf - Sociedade Brasileira de Geofísica

Este texto foi preparado para a apresentação no VII Simpósio Brasileiro de Geofísica, Ouro Preto, 25 a 27 de outubro de 2016. Seu conteúdo foi revisado pelo Comitê Técnico do VII SimBGf, mas não necessariamente representa a opinião da SBGf ou de seus associados. É proibida a reprodução total ou parcial deste material para propósitos comerciais sem prévia autorização da SBGf.

\section{Abstract}

This study presents an exploratory workflow performed in a dataset acquired in northern Santos basin, near the Oliva field, southeast of Brazil. The workflow consists of geoscience activities, comprehending mainly the fields of Geology, Geophysics, Petrophysics and Geostatistics applied to the available dataset (2D seismic lines in Strike and Dip directions, 3D seismic volume and data obtained from eight wells drilled around the area, including stratigraphic markers and well-logs). As results, two opportunities were defined: two leads in the Pre-Salt Play, which were named as, Açaí and Guaraná, respectively. The volumetric analysis establishes a VOIP of $427 \mathrm{MMbbl}$ (P50) for Açaí and 453 MMbbl (P50) for Guaraná structure. Comparisons between the volumetric data obtained during this research and those forecasted by ANP (National Petroleum Agency) and PETROBRAS concerning the pre-salt reservoirs came to assist and enhance the reliability of the present study.

\section{Introdução}

Situada na margem continental do sudeste do Brasil, a Bacia de Santos possui cerca de $350.000 \mathrm{~km}^{2}$, atingindo a cota batimétrica de $3.000 \mathrm{~m}$. Seus limites são definidos ao norte com a bacia de Campos pelo Alto de Cabo Frio, e ao sul com a bacia de Pelotas, pela Plataforma de Florianópolis (Moreira et al., 2007). Nas últimas décadas, diversos autores vêm descrevendo a geologia da bacia de Santos. Em seu trabalho mais recente, Moreira et al. (2007) dividiu a bacia estratigraficamente em quatro unidades litoestratigráficas principais: Gr. Guaratiba, Gr. Camburiú, Gr. Frade e Gr. Itamambuca. Estas encontram-se sobrejacentes ao embasamento cristalino, composto por granitos e gnaisses pré-cambrianos pertencentes à Faixa Ribeira (Cainelli et al., 1999).

As primeiras campanhas exploratórias na bacia tiveram início no final da década de 60 , com a realização das primeiras aquisições sísmicas. O início da fase de investigação direta se deu com a perfuração do poço pioneiro 1-PRS-1, perfurado em 1970. No final da década de 70 a Pecten obteve a primeira descoberta da bacia, a acumulação de gás do campo de Merluza. Com a evolução das campanhas exploratórias e obtenção de maiores volumes de dados sísmicos e de poço, outras jazidas em reservatórios da sequência pós-salífera, de pequeno porte, foram descobertas e estão em produção na bacia (Coral, Estrela do Mar, Tubarão, etc.). Nos últimos anos, a bacia de Santos passou a ser alvo de investimentos mais significativos que culminaram na descoberta de campos de óleo pesado e gás natural em reservatórios pós-salíferos, assim como na descoberta de acumulações de óleo leve na sequência pré-sal. As primeiras compreendem principalmente acumulações em reservatórios turbidíticos, como aqueles encontrados nos campos de Atlanta, Oliva. A partir de 2006 foram anunciadas descobertas em reservatórios da sequência pré-sal, caracterizadas por grandes acumulações como no Campo de Lula e Libra, além de outras como Búzios, Lapa, Júpiter, Sapinhoá. Figura 1

Segundo Carminatti et al., (2008) e Gomes et al., (2008), o desenvolvimento de extensas acumulações nessa seção deve-se a quatro fatores principais: (1) presença de rochas geradoras prolíficas e maturas; (2) presença de altos estruturais intrabacinais importantes para 0 trapeamento e focalização do hidrocarboneto; (3) desenvolvimento de reservatórios carbonáticos microbiais com boa permo-porosidade e (4) presença de espessa camada de evaporitos que atuam como selo das acumulações.

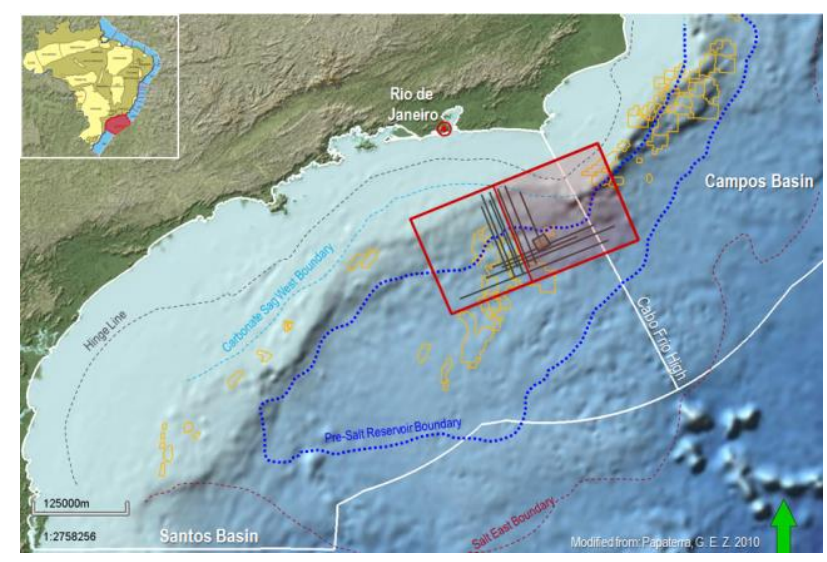

Figura 1 - Mapa de localização da bacia de Santos e polígono de dados. Modificado de Papaterra 2010 e BDEP Webmaps (ANP).

\section{Metodologia}

Os objetivos principais deste trabalho envolveram: Executar uma das metodologias aplicadas na indústria de Óleo \& Gás para a detecção e avaliação de possíveis Leads, Prospectos e Plays exploratórios na bacia de Santos para o intervalo referente ao Pré-Sal, e buscar transpor os desafios e limitações dos dados sísmicos disponíveis na obtenção de uma avaliação exploratória correlacionável da área. 
O pacote de dados disponíveis para a realização do presente estudo foi disponibilizado pela Agência Nacional do Petróleo (ANP) através do seu banco de dados (BDEP). O mesmo compreende um (1) volume sísmico 3D, doze (12) linhas 2D e 8 poços. Após o carregamento dos dados, foi feita a correlação das informações de poços e sísmica, definindo qual seção expressa as características sísmicas mais correlacionáveis com as unidades litoestratigráficas constatadas nos poços. Tal correlação é obtida através da construção do sismograma sintético, o qual é oriundo da modelagem das respostas sísmicas referentes as diferentes unidades litológicas atravessadas pelos poços. A partir desta correlação dos dados inicia-se 0 mapeamento dos refletores sísmicos e o uso de atributos sísmicos no qual abrange o conjunto de metodologias com base no dado sísmico para instrumento de análise e descrição da relação estrutural e estratigráfica da geologia em subsuperfície atuantes na região de estudo. Como parte importante do estudo de identificação dos possíveis leads e prospectos na interpretação sísmica, a etapa da criação do modelo de velocidades e a conversão das superfícies interpretadas nas sísmicas em tempo para a profundidade real observada pelos poços e seus marcadores litológicos.

Devido à incerteza, maior ou menor, mas sempre presente de qualquer método de conversão e por consequentes distorções que podem ocorrer nos dados convertidos, tendo como exemplos; modificações em fechamentos de estruturas e distorções causadas pela presença de camadas espessas de evaporitos, como o pull-up, fazem que esta seja a etapa mais crítica e sujeita a erros da interpretação e infelizmente impactando a estimativa de reservas calculadas. Paralelamente, a etapa de caracterização petrofísica do reservatório do Pré-Sal devido ao fato dos poços não atingirem essa seção, os dados foram obtidos através de pesquisa bibliográfica de análogos.

\section{Resultados}

Após o mapeamento dos horizontes sísmicos nas linhas 2D e no volume 3D sísmico, foi possível gerar as superfícies em tempo para cada refletor e sua visualização tridimensional das estruturas interpretadas nesta região e consequentemente a sua conversão para profundidade conforme discutido anteriormente. Dado as restrições das informações disponíveis para o objetivo deste trabalho (poços que não atravessam os intervalos de interesse, na área do pré-sal, ausência de velocidades sísmicas oriundas do processamento sísmico, dados de petrofísica, e etc), mostrou-se necessária a busca por informações análogas na literatura para correlacionar os parâmetros necessários as análises nos intervalos supracitados. Figura 2

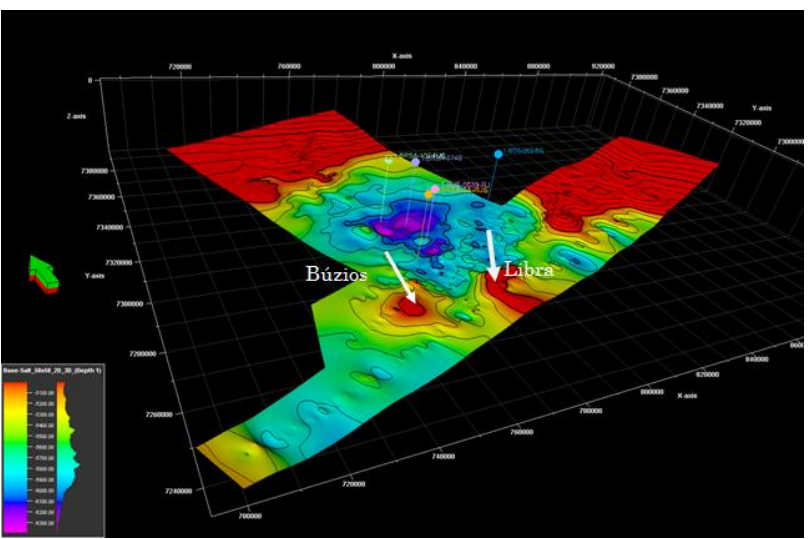

Figura 2 - Superfície referente ao reservatório pré-sal, nas linhas $2 D$ e $3 D$, convertido em Profundidade. Na imagem é possível correlacionar as estruturas de Búzios e Libra (setas).

A partir do modelo de velocidades criado, converteu-se as superfícies e sísmicas existentes, (linhas 2D e volume 3D) para profundidade, e assim começar a etapa de estimativas volumétricas nas estruturas visualizadas que possam caracterizar possíveis leads/prospectos a serem avaliados. Com a concepção do modelo de deposição e a caracterização das possíveis acumulações, foram definidos dois leads, denominados Açaí e Guaraná, no Play do Pré-Sal, figura 3. Os leads Açaí e Guaraná envolvem os reservatórios microbiais/estromatolíticos e coquinhas da Fm. Barra Velha, de idade Aptiana. Para a análise do Play Pré-sal, foram disponibilizados apenas dados de sísmica, sendo assim, necessária a utilização dos dados de campos análogos como Búzios, Libra, Lula, lara, Júpiter para consolidar informações dos poços, como litologia encontrada, parâmetros petrofísicos e de fluido adquiridos em testes de formação feitos nesses campos. Tal conjunto, esclareceu e norteou a definição de possíveis áreas a serem exploradas. Para uma análise mais ampla dos prospectos encontrados, os valores utilizados de porosidade efetiva, saturação de água e Net To Gross para os cálculos de VOIP levaram em conta sua moda, valores mínimos e máximos (Tabela 1). Essa metodologia visa abranger a grande variabilidade de condições permo-porosas apresentadas por esses reservatórios, assim como mitigar os riscos envolvidos nesses prospectos.

Tabela 1 - Parâmetros petrofísicos utilizados como suporte para o cálculo de VOIP. Modificado de Gaffney, Cline \& Associates. ANP, 2010.

\begin{tabular}{|l|c|c|c|}
\cline { 2 - 4 } \multicolumn{1}{c|}{} & Min (\%) & Moda (\%) & $\operatorname{Max}(\%)$ \\
\hline $\boldsymbol{\phi}(\%)$ & 4 & 10 & 14 \\
\hline SW & 15 & 21 & 31 \\
\hline NTG & 41 & 76 & 93 \\
\hline
\end{tabular}




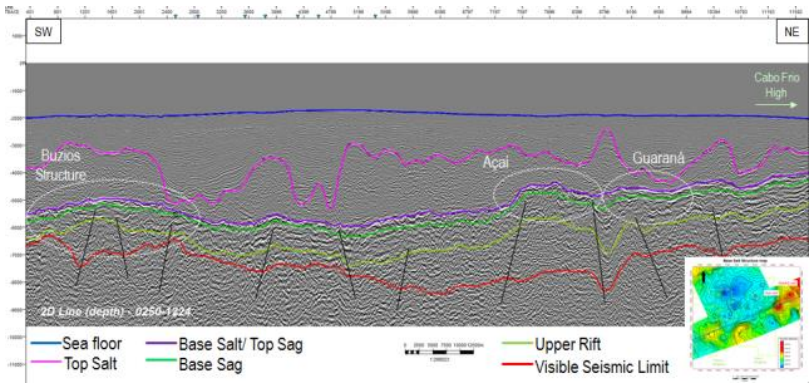

Figura 3 - Seção sísmica representativa dos Leads Açaí e Guaraná (em profundidade).

O Play do pré-sal consiste em reservatórios de calcário Aptiano da Formação Barra Velha (microbiolitos e estromatólitos depositados em condições marítimas transicionais, hipersalinas, geralmente referidas como "seção SAG" sismicamente distinta), algumas vezes calçada por uma seção adicional de reservatório em calcário, a Formação Itapema (coquinas ou bancos de conchas depositados em condições lacustres no topo da seção sin-rifte (Gaffney, Cline \& Associates. 2010). Com o horizonte sísmico da Base do Sal/ Topo do SAG convertido foi possível delimitar duas regiões de interesse, estruturadas na base dos evaporitos, com fechamento quaquaversal, que são os leads de Açaí e Guaraná, com áreas de 47,6 e $68,5 \quad \mathrm{~km}^{2}$, respectivamente. $\mathrm{Na}$ etapa da avaliação de riscos e volumetria, foi feita uma intensa revisão na bibliografia digital publicada na internet, buscando o histórico de descobertas, de produção e dos poços disponíveis para fomentar os valores dos parâmetros petrofísicos e de fluido a serem utilizados na estimativa dos volumes de óleo in place e recuperáveis para essas propostas exploratórias. Tabela 2.

Tabela 2 - Volumes in place e recuperáveis do Lead Açaí e Guaraná.

\begin{tabular}{|c|c|c|c|c|}
\hline Volumes & Lead & P90 & P50 & P10 \\
\hline VOIP, MMbbl & \multirow[b]{2}{*}{ Açaí } & 184 & 427 & 793 \\
\hline $\begin{array}{l}\text { Volume } \\
\text { Recuperável, } \\
\text { MMbbl }\end{array}$ & & 30 & 78 & 162 \\
\hline PG (sucesso) & $29 \%$ & & & \\
\hline VOIP, MMbbl & \multirow[b]{2}{*}{ Guaraná } & 244 & 453 & 785 \\
\hline $\begin{array}{l}\text { Volume } \\
\text { Recuperável, } \\
\text { MMbbl }\end{array}$ & & 36 & 78 & 157 \\
\hline PG (sucesso) & $24 \%$ & & & \\
\hline
\end{tabular}

Além de uma análise individual para os leads de Açaí e Guaraná, foi feita uma análise de risco considerando um cenário onde os dois ocorressem como sendo uma só estrutura, sendo estabelecida máxima correlação dos riscos envolvendo a ocorrência da trapa, do selo e da geração. E foram obtidos os seguintes volumes, como mostra a Tabela 3 abaixo:
Tabela 3 - Volumes in place e recuperáveis dos leads Açaí e Guaraná, caso fossem descobertos e tivessem relação de dependência.

\begin{tabular}{|l|c|c|c|c|}
\hline Volumes & Lead & P90 & P50 & P10 \\
\hline VOIP, MMbbl & Açaí + & 226 & 514 & 1043 \\
\hline $\begin{array}{l}\text { Volume } \\
\begin{array}{l}\text { Recuperável, } \\
\text { MMbbl }\end{array}\end{array}$ & \begin{tabular}{c} 
Guaraná \\
\hline
\end{tabular} & 35 & 92 & 211 \\
\hline
\end{tabular}

\section{Discussão e Conclusões}

$\mathrm{Na}$ área de estudo foram avaliadas duas acumulações na seção Pré-Sal (leads Guaraná e Açaí) cujos os volumes encontram-se listados nas Tabela 2 e 3 . As acumulações da fase Sag estão associadas aos altos estruturais intrabacinais gerados durante a Fase Rifte, os quais atuam no trapeamento e focalização do hidrocarboneto. Para o lead Açaí e Guaraná foram obtidas chances de sucesso (PG) de aproximadamente $29 \%$ e $24 \%$, respectivamente. Devido à baixa qualidade dos atuais levantamentos Pós-migrados utilizados no presente trabalho, torna-se necessária uma melhor avaliação da mesma utilizando dados de aquisições sísmicas com emprego de técnicas de processamento atuais PSTM/PSDM, existentes para a área em questão - de maneira a obter um melhor imageamento das estruturas acima e abaixo dos domos de sal, reduzindo os efeitos gerados pela presença de ruídos, múltiplas e artefatos em geral causados pela baixa razão sinal/ruído nos dados sísmicos. Recomenda-se ainda a utilização do campo de velocidade obtido de um centro de processamento para que a conversão em profundidade seja mais coerente com a geologia atuante na região, com o controle dos parâmetros a partir dos poços presentes na área.

Um novo levantamento sísmico requisitado pela ANP encontra-se em andamento, abrangendo parte da área estudada e estendendo-se ao longo do Alto de Cabo Frio até a bacia de Campos. Este levantamento vem a confirmar o alto interesse exploratório dessa região e confirmará com maior exatidão a disposição das estruturas presentes na seção Pré-Sal. Outro fator positivo para o desenvolvimento de prospectos nessa região deve-se a presença de uma ampla infraestrutura de gasodutos e portos já instalados, a qual auxiliariam no escoamento de uma possível produção. Petersohn et al., (2013) e Abelha., (2015). Figura 4. 

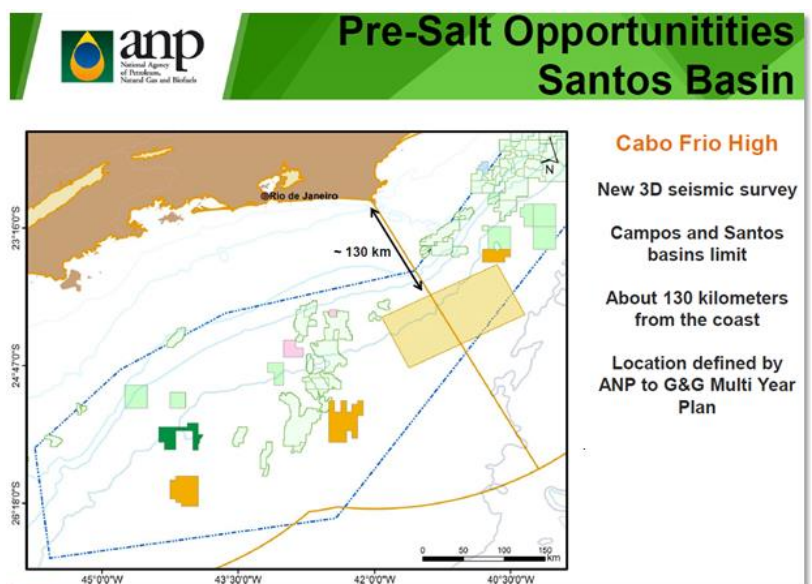

\section{anp Brasil I Infraestruture and PRE-SALT $=$ Operational Conditions}

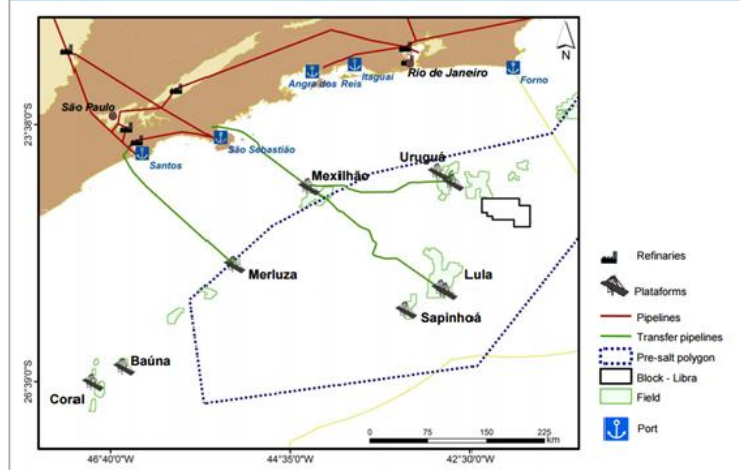

Figura 4 - Oportunidades e infraestrutura de desenvolvimento próximo à área de estudo. Modificado de Petershon 2013 e Abelha 2015.

\section{Agradecimentos}

Os autores gostariam de agradecer ao convênio entre a Universidade do Estado do Rio de Janeiro (UERJ), aos coordenadores e professores envolvidos, a Statoil do Brasil Óleo e Gás e Agência Nacional do Petróleo (ANP) que tornaram possível a realização deste estudo.

\section{Referências}

Abelha, M. F. 2015 Brazilian Petroleum Conference Carbonates: From Genesis to Production - Rio de Janeiro, Brasil.

Cainelli, C., Mohriak, W. 1999. Some Remarks on the Evolution of Sedimentary Basins along the Eastern Brazilian Continental Margin. Episodes, Vol. 22, n. 3. p. 206- 216.

Carminatti M., Wolff B., Gamboa L.A.P. 2008. New exploratory frontiers in Brazil. In: World Petroleum Congress, 19, Proceedings, $11 \mathrm{p}$.
Gaffney, Cline \& Associates, ANP. 2010. Exame de Avaliação de Dez Descobertas e Prospectos Selecionados no Play do Pré-Sal em Águas Profundas na Bacia de Santos, Brasil.

Gomes, P. O., Kilsdonk, B., Minken, J., Grow, T. \& Barragan, R. 2008. The outer high of the Santos Basin, Southern São Paulo Plateau, Brazil: pre-salt exploration outbreak, paleogeographic setting, and evolution of the syn-rift structures. AAPG CD Abstracts, Cape Town.

Moreira, J.P., Madeira, C.V., Gil, J.A., Machado, M.A.P. 2007. Bacia de Santos. Boletim de Geociências da Petrobrás, v.15, n.2, p.531-549.

Papaterra, G. E. Z. 2010. Pré-sal: Conceituação Geológica sobre uma Nova Fronteira Exploratória no Brasil. Dissertação de mestrado. Instituto de Geociências, Universidade Federal do Rio de Janeiro.

Petersohn, E., Abelha, M., Pedrosa, L. 2013. Seminário Técnico Ambiental, apresentação Brasil Pré-sal, Libra Rio de Janeiro, Brasil. 